\title{
Restoration of Blurred Image Using Joint Statistical Modeling in a Space-Transform Domain
}

\author{
Mr Pushpendra Singh ${ }^{1}$, .Prof (Dr) R L Sharma ${ }^{2}$ \\ ${ }^{1}$ M.Tech Student, Noida International University, \\ ${ }^{2}$.Professor \& Head Department Of Electronics \& Communication Engineering, Noida International \\ University, Greater Noida,Uttar Pradesh,(India).
}

\begin{abstract}
This paper presents a completely unique strategy for accurate image restoration by characterizing each native smoothness and nonlocal self-similarity of natural pictures during a unified statistical manner. the most contributions area unit three-fold. First, from the angle of image statistics, a joint statistical modeling (JSM) in an accommodative hybrid space-transform domain is established, that offers a strong\} mechanism of mixing native smoothness and nonlocal self-similarity at the same time to make sure a a lot of reliable and robust estimation. Second, a replacement variety of minimization purposeful for finding the image inverse drawback is developed using JSM underneath a regularization-based framework. Finally, so as to form JSM tractable and robust, a replacement Split Bregman-based algorithmic program is developed to expeditiously solve the on top of severely underdetermined inverse drawback associated with theoretical proof of convergence. intensive experiments on image inpainting, image deburring, and mixed mathematician and saltand pepper noise removal applications verify the effectiveness of the proposed algorithmic program.
\end{abstract}

Keywords: Inpainting ,JSM, Restoration, Image Blur, Statistical Modeling.

\section{Introduction}

Concept of image restoration started in $1950^{\circ} \mathrm{s}$. Image restoration is based on the concept to improve the quality of an image through knowledge of the image formation process or physical process which led to its formation. The purpose of image restoration is to "undo" defects or damage which degrade an image. Degradation occurs due to different reasons such as motion blur, noise, and miss-focus of camera. There are various types of blur model. In cases of motion blur, it is possible to obtain a very good estimate of the actual blurring function and remove the blur to restore the original image. a joint statistical modeling (JSM) in an adaptive hybrid space-transform domain is established, which offers a powerful mechanism of combining local smoothness and nonlocal self-similarity simultaneously to ensure a more reliable and robust estimation. Second, a new form of minimization functional for solving the image inverse problem is formulated using JSM under a regularization-based framework The nonlocal self-similarity depicts the repetitiveness of higher level patterns (e.g., textures and structures) globally positioned in images, which is first utilized to synthesize textures and fill in holes in images nonlocal self-similarity of natural images in a unified statistical manner. Image restoration is different process from image enhancement. In image enhancement the image features are extracted instead of restoration of degraded image. Image enhancement is the process in which the degraded image is handled and the appearance of the image by visual is improved. It is the subjective process and increases contrast of image but image restoration is a more and more objective process than image enhancement. Image restoration problems can be measured very precisely, where as enhancement process is difficult to represent in mathematical form. There are several application of image restoration like, legal investigations, scientific purpose, film making and archival, image and video coding and decoding and photography purpose [1]. The main application of image restoration i.e. image reconstruction is in radio astronomy,

\section{Aim Of The Project}

The main aim of this project is to produce original high quality image from high-fidelity image restoration by characterizing both local smoothness and nonlocal self-similarity of natural images in a unified statistical manner.

\section{Literature Survey}

One of the related areas that has seen a recent surge of interest is the area of image processing. With the rise of digital cameras, scanners, and digital imagery, it is more important than ever to have good methods for processing this imagery. The digital images could be distorted or have noise which needs to be removed. Some researchers are interested in recovering important features contained in the image. For example, they may want to recover the location of buildings in satellite imagery or identify objects that are blocking a path for a mobile 
robot. Given that many images require massive amounts of storage space, we need to consider methods by which to compress the imagery in order to save on storage costs.

A good example of the problems encountered with digital imagery is given by NASA's Deep Impact mission spacecraft. This satellite was sent to study the innards of a comet, but one of the instrument cameras was not properly calibrated before it was sent into space. The result was all the imagery returned to earth was blurry and all the images would have to be corrected after the data was returned. In image restoration, a distorted image is restored to its' original form. This distortion is typically caused by noise in transmission, lens calibration, motion of the camera, or age of the original source of the image.

As a fundamental problem in the field of image processing,image restoration has been extensively studied in the past two decades [1]. It aims to reconstruct the original high quality image $\boldsymbol{x}$ from its degraded observed version $\boldsymbol{y}$, whichis a typical ill-posed linear inverse problem and can be generally formulated as

$$
\boldsymbol{y}=\boldsymbol{H} \boldsymbol{x}+\boldsymbol{n},
$$

where $\boldsymbol{x}, \boldsymbol{y}$ are lexicographically stacked representations of the original image and the degraded image,respectively, $\boldsymbol{H}$ is a matrix representing a non-invertible linear degradation operator and $\boldsymbol{n}$ is usually additive Gaussian white noise. When $\boldsymbol{H}$ is identity,

\section{(a) Total Variation (TV)}

Total variation, it is a regularization process, most often used in digital image processing, that has applications in noise removal. It is based on the principle that signals with excessive and possibly spurious detail have high total variation, that is, the integral of the absolute gradient of the signal is high. According to this principle, reducing the total variation of the signal subject to it being a close match to the original signal,

\section{(b) Mumford-Shah (MS) model}

The Mumford-Shah model is a functional that is used to establish an optimality criterion for segmenting an image into sub-regions. An image is modeled as a piecewise-smooth function. The functional penalizes the distance between the model and the input image, the lack of smoothness of the model within the sub-regions, and the length of the boundaries of the sub-regions. By minimizing the functional one may compute the best image segmentation. Image segmentation is a hot topic of research given its applicability as a pre-processing technique in many image understanding applications. This Mumford-Shah model describes the image segmentation. The mathematical framework and the main features of the model are sketched along with the procedure leading from the analytical expression of the model to its practical implementation.

\section{Deblurring}

One of the most common artifacts in digital photography is motion blur caused by camera shake. In many situations there simply is not enough light to avoid using a long shutter speed, and the inevitable result is that many of our snapshots come out blurry and disappointing. Recovering an un-blurred image from a single, motion-blurred photograph has long been a fundamental research problem in digital imaging. If one assumes that the blur kernel - or point spread function (PSF) - is shift-invariant, the problem reduces to that of image deconvolution. Image deconvolution can be further separated into the blind and non-blind cases.

\section{Inpainting}

Inpainting is the process of reconstructing lost or deteriorated parts of images and videos.For instance,in the museum world, in the case of a valuable painting, this task would be carried out by a skilled art conservator or art restorer. In the digital world, inpainting (also known as image interpolation or video interpolation) refers to the application of sophisticated algorithms to replace lost or corrupted parts of the image data (mainly small regions or to remove small defects).Inpainting, the technique of modifying an image in an undetectable form, is as ancient as art itself. The goals and applications of inpainting are numerous, from the restoration of damaged paintings and photographs to theremoval/replacement of selected objects. In this paper, we introduce a novel algorithm for digital inpainting of still images that attempts to replicate the basic techniques used by professional restorators.

\section{Noise Removal}

Is to decide that features that exist on a very small scale in the image are noise, and that removing these while maintaining larger features might help "clean things up".One well-traveled approach is to smooth the image. Denoise images. The main problem faced during diagnosis is the noise introduced due to the consequence of the coherent nature of the image capture. In image processing Imagine an image with noise. For example, the image on the left below is a corrupted binary (black and white) image of some letters; $60 \%$ of the pixels are thrown away and replaced by random gray values ranging from black to white.One goal in image restoration is to remove the noise from the image in such a way that the "original"image is discernible. Of 
course, "noise" is in the eye of the beholder; removing the "noise" from a Jackson Pollack painting would considerably reduce its value. Nonetheless, one approach applications, linear filters tend to blur the edges and do not remove Gaussian and mixed Gaussian impulse noise effectively. Inherently noise removal from image introduces blurring in many cases. These noises corrupt the image and often lead to incorrect diagnosis.

\section{Proposed Joint Statistical Modeling In Spacetransform Domain}

The ill-posed nature of image inverse problems, prior knowledge about natural images is usually employed, namely image properties, which essentially play a key role in achieving high-quality images two types of popular image properties are considered, namely local smoothness and nonlocal self-similarity, The challenge is how to characterize and formulate these two image properties mathematically. Note that different formulations of these two properties will lead to different results.
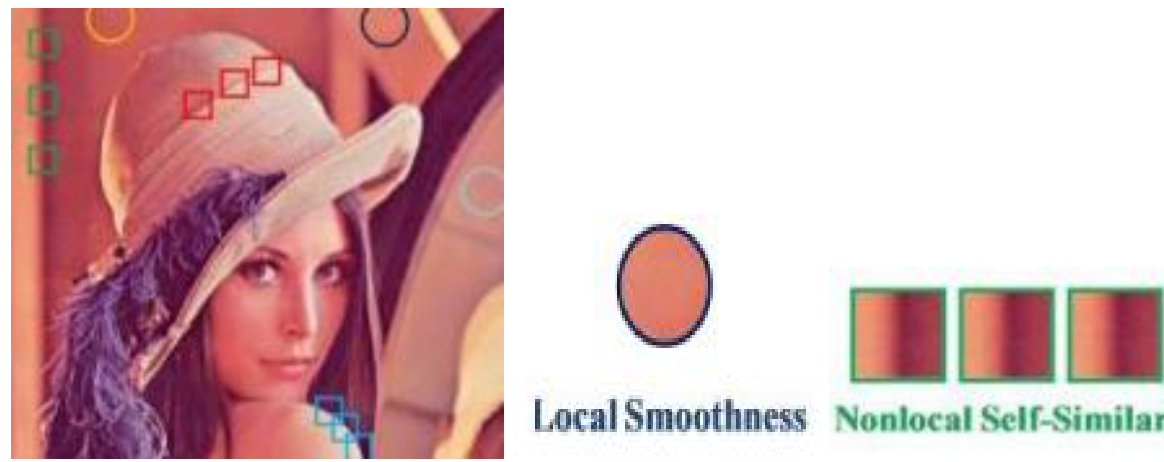

\section{Local Smoothness Nonlocal Self-Similarity}

Fig. 1. Illustrations for local smoothness and nonlocal self-similarity of natural images.

In this study, we characterize these two properties from the perspective of image statistics and propose a joint statistical modeling (JSM) for high fidelity of image restoration in an adaptive hybrid spacetransform domain. Specifically, JSM is established by merging two complementary models- local statistical modeling (LSM) in two-dimensional space domain and nonlocal statistical modeling (NLSM) in three-dimensional transform domain.

\section{(i) Local Statistical Modeling for Smoothness in Space Domain}

Local smoothness describes the closeness of neighboring pixels in the two-dimensional space domain of images, which means the intensities of the neighboring pixels are quite similar. there exist many models. Here, we mathematically formulate a local statistical modeling for smoothness in two-dimensional space domain. From the view of statistics, a natural image is preferred when its responses for a set of high passing filters are as small as possible which intuitively implies that images are locally smooth and their derivatives are close to zero.

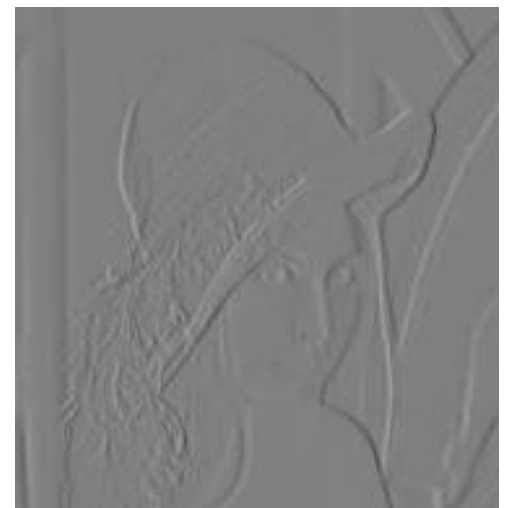

(a)

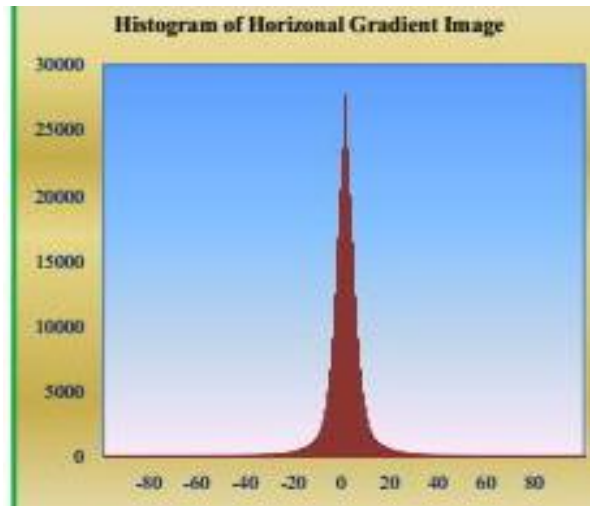

(b)

Fig. 2. Illustrations for local statistical modeling for smoothness in space domain at pixel level. (a) Gradient picture in horizontal direction of image Lena; (b) Distribution of horizontal gradient picture of Lena,

Note that $\Psi$ LSM has the same expression as anisotropic TV defined and can be regarded as a statistical interpretation of anisotropic TV. It is important to emphasize that local statistical modeling is only used for characterizing the property of image smoothness. The regularization term has the advantages of convex 
optimization and low computational complexity. There is no need to design a very complex regularization term, since the task of retaining the sharp edges and recovering the fine textures will be accomplished by the following nonlocal statistical modeling.

\section{Experimental Results}
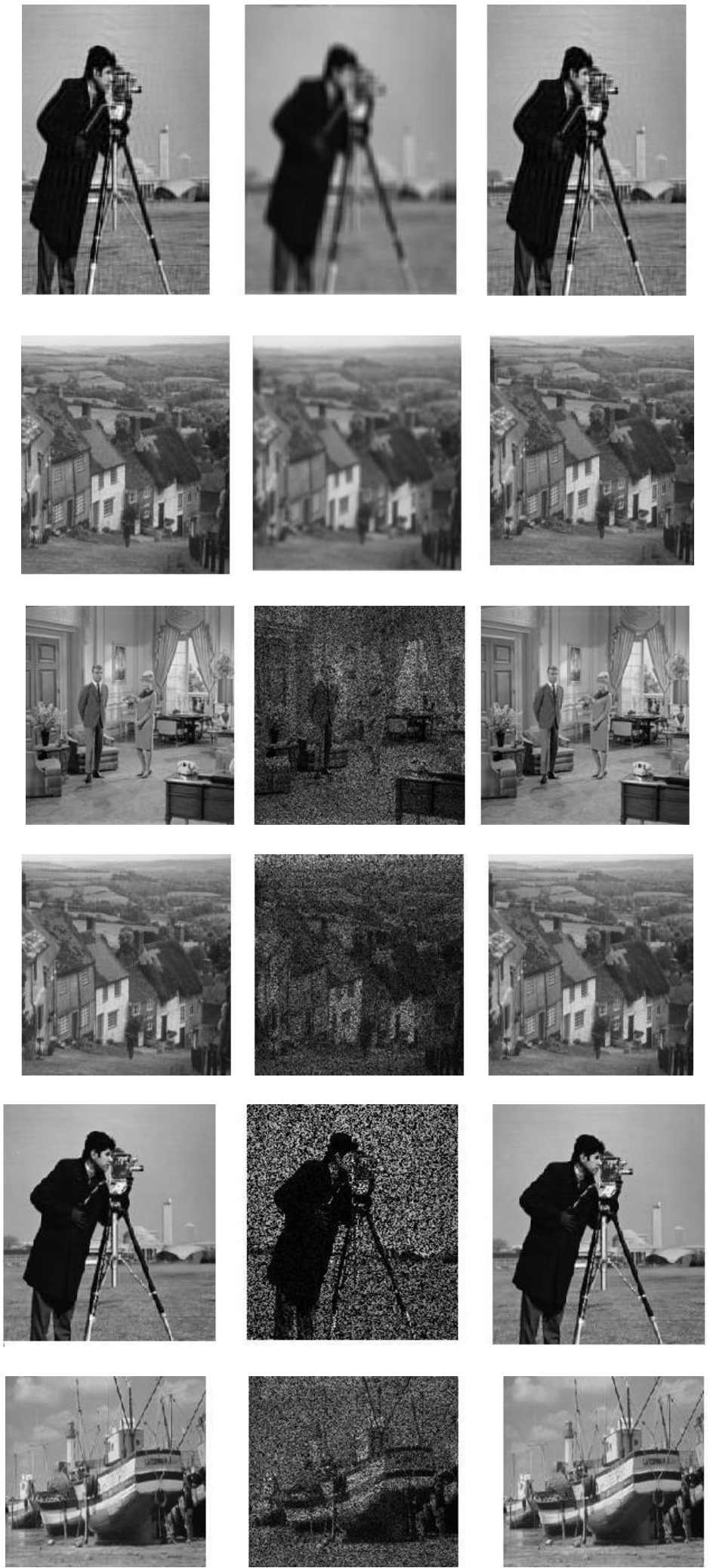


\section{Conclusions And Future Scope}

The experimental results are presented to evaluate the performance of the proposed algorithm, which is compared with many existing methods. We apply our algorithm to the applications of image inpainting, image deblurring, and mixed Gaussian plus salt-and-pepper noise removal. To evaluate the quality of image reconstruction, in addition to PSNR, which is used to evaluate the objective image quality.

A novel algorithm for high-quality image restoration using the joint statistical modeling in a spacetransform domain is proposed, which efficiently characterizes the intrinsic properties of local smoothness and nonlocal self-similarity of natural images from the perspective of statistics at the same time.

Future work includes the investigation of the statistics for natural images at multiple scales and orientations and the extensions on a variety of applications, such as image deblurring with mixed Gaussian and impulse noise and video restoration tasks.

\section{Acknowledgements.}

I thank my guide Prof (DR).R L SHARMA for supporting my research work in Restoration of Blurred Image Using Joint Statistical Modeling in a Space-Transform Domain.

\section{References}

[1]. M. R. Banham and A. K. Katsaggelos, "Digital image restora-tion,” IEEE Trans. Signal Processing Mag., vol. 14, no. 2, pp. 24-41, Mar. 1997

[2]. A. Chambolle, “An algorithm for total variation minimization and applications,” J. Math.Imaging Vis., vol. 20, pp. 89-97, 2004

[3]. Y. Chen, K. Liu, "Image Denoising Games," IEEE Trans. on Circuits and Systems for Video Technology, vol. 23, no. 10, pp. 17041716, Oct. 2013.

[4]. J. Zhang, D. Zhao, C. Zhao, R. Xiong, S. Ma and W. Gao, "Image compressive sensing recovery via collaborative sparsity," IEEE J. on Emerging and Selected Topics in Circuits and Systems, vol. 2, no. 3, pp. 380-391, Sep. 2012.

[5]. X. Zhang, R. Xiong, X. Fan, S. Ma and W. Gao, "Compression artifact reduction by overlapped-block transform coefficient estimation with block similarity,” IEEE Trans. on Image Process., vol. 22, no. 12, pp. 4613-4626, Dec. 2013.

[6]. W. Dong, L. Zhang, G. Shi, and X. Wu, "Image deblurring and super resolution by adaptive sparse domain selection and adaptive regularization,” IEEE Trans. Image Jul. 2011. 